\title{
Associations between pulsars and supernova remnants
}

\author{
B.M. Gaensler and Simon Johnston \\ Research Centre for Theoretical Astrophysics, University of Sydney, \\ NSW 2006, Australia
}

\section{Introduction}

We have performed Monte Carlo simulations of the Galactic supernova (SN), supernova remnant (SNR) and pulsar (PSR) populations, and find that even if every SN forms a fast-spinning pulsar, the number of shell type remnants which are expected to have an associated pulsar is even less than observed. We conclude that most shell SNRs will not have a detectable pulsar associated with them, and that many claimed associations are merely geometric projection.

\section{The Model}

In. our recent paper (Gaensler \& Johnston 1995c), we assume that every SN produces a shell SNR and a pulsar, with a Galactic birth rate of one per 85 years. SNe are distributed throughout the Galaxy in a thin disk (Johnston 1994). We then model:

- SNR evolution

- Pulsar evolution

- Selection effects in SNR searches

- Selection effects in pulsar searches

\section{Results of the Simulation}

The number of PSR/SNR associations obtained in the simulation is significantly less than that observed. We therefore conclude that:

- A large fraction of SNe form both SNRs and pulsars

- In very few SNRs will the associated pulsar be detectable

- Roughly half of all associations are geometric coincidence

Even after using the revised pulsar velocity law of Lyne \& Lorimer (1994), we find that in $98 \%$ of simulated associations younger than $25000 \mathrm{yr}$, the pulsar is well inside the SNR's boundaries. Thus claimed associations involving a young pulsar located outside an SNR are probably spurious (Gaensler \& Johnston 1995a). 
Of the 15 claimed associations involving a pulsar having $\tau<10^{5} \mathrm{yr}$ (where $\tau=P / 2 \dot{P}$ ), only five can be said to be well-established (those containing PSRs $\mathrm{B} 0531+21, \mathrm{~B} 0540-69, \mathrm{~B} 0833-45, \mathrm{~B} 1853+01$ and $\mathrm{B} 2334+61)$. We consider the following associations the most likely to be geometric; in all five cases, the pulsar is near the edge of or outside the SNR:

- SNR IC 443 \& PSR B0611+22 (Weisberg et al 1980)

- SNR W 30 \& PSR J1803-2137 (Frail et al 1994a)

- SNR MSH 16-51 \& PSR J1614-5047 (Gaensler \& Johnston 1995a)

- SNR G343.1-2.3 \& PSR B1706-44 (Frail et al 1994b; Nicastro et al 1996)

- SNR W 28 \& PSR J1801-23 (Kaspi et al 1993)

SNRs can be long-lived - some may be as old as $5 \times 10^{5}$ yr. In associations involving such SNRs, the pulsar can be located well outside the remnant. Thus there exist possible associations between pulsars with $\tau \sim 10^{5} \mathrm{yr}$ and adjacent remnants, e.g. PSR B1830-08 and SNR W 41 (Gaensler \& Johnston 1995b).

Finally, the distribution of detectable SNRs in Galactic longitude can only be explained if SNRs far from the Galactic centre are generally larger and older than those closer in.

\section{Further Work}

- HI measurements on SNR G320.4-1.2 to determine if the distance to the various components is consistent with that to the pulsar B1509-58.

- High resolution imaging of SNR G308.8-0.1 to determine if it is interacting with PSR B1338-62.

- High resolution imaging of PSR J1614-5047 in the radio and in X-rays to search for a possible plerion around the pulsar.

- Simulations of filled-centre or "plerionic" remnants

\section{References}

Frail, D. A., Kassim, N. E., Weiler, K. W. 1994a, AJ, 107, 1120

Frail, D. A., Goss, W. M., Whiteoak, J. B. Z. 1994b, ApJ, 437, 781

Gaensler, B. M., Johnston, S. 1995a, PubASA, 12, 76

Gaensler, B. M., Johnston, S. 1995b, MNRAS, 275, L73

Gaensler, B. M., Johnston, S. 1995c, MNRAS, 277, 1243

Johnston, S. 1994, MNRAS, 268, 595

Kaspi, V. M., Lyne, A. G., Manchester, R. N., Johnston, S., D’Amico, N., Shemar, S. L. 1993, ApJ, 409, L57

Lyne, A. G., Lorimer, D. R. 1994, Nature, 369, 127

Nicastro, L., Johnston, S., Koribalski, B. 1996, A\&A, 306, 49

Weisberg, J. M., Rankin, J. M., Boriakoff, V. 1980, A\&A, 88, 84 